Mathematical Research Letters 1, 765-768 (1994)

\title{
A COUNTER EXAMPLE IN THE ERGODIC THEORY OF EXPANDING MARKOV MAPS OF THE INTERVAL
}

\author{
A. G. Abercrombie And R. NAir
}

\begin{abstract}
A Bstract. A theorem popularly called the folklore theorem gives conditions under which an expanding Markov map of the unit interval is known to preserve a measure equivalent to Lebesgue measure, with respect to which it is ergodic and even exact. There is some uncertainty as to how much the hypotheses of this theorem can be weakened. We provide a counter example to one possible strengthening erroneously stated in $[\mathrm{Ma}]$.
\end{abstract}

Let $T$ be a self map of $[0,1]$ and let $\mathcal{P}_{0}=\{P(j): j \in \Lambda\}$ be a partition of $[0,1]$ into open intervals, disregarding a set of Lebesgue measure zero. We may for instance take $\Lambda$ to be $\{1,2, \cdots, n\}(n=1,2, \cdots)$ or we may take $\Lambda$ to be the natural numbers.

Let $\lambda$ denote Lebesgue measure. We say the map $T$ is an expanding Markov map with partition $\mathcal{P}_{0}$ if the following conditions hold :

(i) for each $j$ in $\Lambda$ there exists $\Lambda_{j} \subset \Lambda$ such that

$$
T(P(j))=\operatorname{int} \overline{\bigcup_{i \in \Lambda_{j}} P(i)}
$$

(ii) we have

$$
\inf _{j \in \Lambda} \lambda(T(P(j))>0
$$

(iii) the derivative $T^{\prime}$ of $T$ is defined and $\frac{1}{T^{\prime}}$ is bounded on $\cup_{j \in \Lambda} P(j)$;

(iv) there exists $\beta>1$ such that $\left(T^{n}\right)^{\prime} \gg \beta^{n}$ whenever it exists and

(v) there exists $\gamma$ in $(0,1)$ such that

$$
\left|1-\frac{T^{\prime}(x)}{T^{\prime}(y)}\right| \ll|x-y|^{\gamma},
$$

for $(x, y)$ in $\operatorname{diag}\left(\mathcal{P}_{0}\right)$.

The following statement has become known as the folklore theorem [Ad]. See also $[\mathrm{KS}]$ and $[\mathrm{LY}]$.

Received October 7, 1994. 
Theorem. If $T$ is an expanding Markov map then there exists a probability measure $\mu$ on $[0,1]$ preserved by $T$ (that is $\mu\left(T^{-1}(A)\right)=\mu(A)$ for each element $A$ of the Borel $\sigma$-algebra $\mathcal{A}$ of $[0,1])$, which is equivalent to Lebesgue measure on $[0,1]$.

The dynamical system $([0,1], \mathcal{A}, \mu, T)$ is ergodic and in fact exact. It has been claimed (see Theorem 1.2 page 168 of [Ma]) that the theorem holds even for $T$ satisfying the following weaker hypothesis in place of (ii):

(ii)' there exists an integer $m$ such that for each $i, j$ in $\Lambda$ we have

$$
\lambda\left(P(i) \cap T^{-m}(P(j))\right)>0 .
$$

However the argument in [Ma] tacitly assumes (ii) (see the proof of Lemma III 1.5 on p.174). In this note we present an example of a map showing that (ii)' does not imply (ii) even in the presence of (i), (iii), (iv) and (v) and also that the folklore theorem fails when (ii) is replaced by (ii)'.

The example. We take $\mathcal{P}_{0}$ to be the collection of intervals $P(n)=\left(\frac{1}{n+1}, \frac{1}{n}\right)$ for $n=1,2, \cdots$. We take $T$ to be linear and increasing on each interval of $\mathcal{P}_{0}$ so $T$ will be defined (disregarding a countable set of values) as soon as we have specified $T(P(n))$ for each $n$. We set

$$
T(P(n))= \begin{cases}(0,1), & \text { if } n+1 \text { is a power of } 2 \\ \left(0, \frac{2}{n(n+1)}\right), & \text { otherwise. }\end{cases}
$$

Since either $n$ or $n+1$ is even $\frac{2}{n(n+1)}$ is always the reciprocal of an integer so if $n+1$ is not a power of 2 we have

$$
T(P(n))=\operatorname{int} \varlimsup_{j \geq \frac{n(n+1)}{2}-1} P(j) .
$$

This means that $T$ satisfies (i). Clearly for each $n, T(P(n))$ contains $P\left(2^{k}-\right.$ 1) for all sufficiently large $k$, so $T^{2}(P(n))=(0,1)$ and $T$ satisfies (ii)' with $m=2$. Conditions (iii) and (v) hold because $T$ is piecewise linear with $T^{\prime} \geq 2$ on each $P(n)$ and condition (iv) follows from the chain rule (take $\beta=2$ and then (iv) holds with implied constant 1). However it is clear that (ii) is not true. 
To see the folklore theorem fails for this choice of $T$, suppose that a measure $\mu$ exists satisfying

$$
\mu\left(T^{-1}(A)\right)=\mu(A)
$$

for each set $A$ in the Borel $\sigma$-algebra $\mathcal{A}$ of $[0,1]$ and also

$$
C_{1} \leq \frac{d \mu}{d \lambda} \leq C_{2}
$$

for some $C_{1}$ and $C_{2}$ in $(0, \infty)$. Then we have $\mu(A) \leq \mu(T(A))$ and also

$$
C_{1} \leq \frac{\mu(A)}{\lambda(A)} \leq C_{2}
$$

whenever $\lambda(A)>0$. For each $k=1,2, \cdots$, write

$$
A_{k}=\left(\frac{1}{2^{k+1}-1}, \frac{1}{2^{k}}\right)
$$

so that $A_{k}$ is (up to a finite set of endpoints) the union of the intervals

$$
P\left(2^{k+1}-2\right), \cdots, P\left(2^{k}\right) .
$$

We have

$$
T\left(A_{k}\right)=T\left(P\left(2^{k}\right)\right)=\left(0, \frac{2}{2^{k}\left(2^{k}+1\right)}\right)
$$

and so

$$
\lambda\left(A_{k}\right)=\frac{2^{k}-1}{2^{2 k+1}-2^{k}}
$$

with

$$
\lambda\left(T\left(A_{k}\right)\right)=\frac{2}{2^{k}\left(2^{k}+1\right)} .
$$

But then using (2) we have

$$
C_{2} \geq \frac{\mu\left(T\left(A_{k}\right)\right)}{\lambda\left(T\left(A_{k}\right)\right)} \geq \frac{\mu\left(A_{k}\right)}{\lambda\left(T\left(A_{k}\right)\right)} \geq C_{1} \frac{\mu\left(A_{k}\right)}{\lambda\left(T\left(A_{k}\right)\right)} \geq 2^{k-4} C_{1},
$$

for each $k$, and this is impossible if both $C_{1}$ and $C_{2}$ are in $(0, \infty)$. Hence (1) fails and therefore no measure satisfying the required conditions can exist. 


\section{References}

[Ad] R.L Adler, F-expansions revisited, Lecture Notes in Math., vol. 318, SpringerVerlag, 1973, pp. 1-5.

[KS] K. Krzyżewski and L. Szlenk, On invariant measures for expanding differentiable mappings, Studia Math. 33 (1968), 83-92.

[LY] A. Lasota and J.A. Yorke, On the existence of invariant measures for piecewise monotone transformations, Trans. Amer. Math. Soc. 186 (1973), 481-488.

[Ma] R. Mañé, Ergodic theory and differentiable dynamics, Springer-Verlag, Ergebnisse der Mathematik und ihrer Grenzgebiete, 3. Folge Band 8, 1987.

Department of Pure Mathematics, University of Liverpool, P.O. Box 147 ,

LiVERPOOL L $693 \mathrm{~B}$ X, U.K.

E-mail address: aa1729@liverpool.ac.uk, nair@liverpool.ac.uk 